\title{
A rare case of extrahepatic biliary mucinous cystic neoplasm in a middle-aged woman: A case report
}

\author{
ANNA PASPALA $^{1}$, NIKOLAOS MACHAIRAS ${ }^{2}$, VASILEIA DAMASKOU ${ }^{3}$, VASILEIA NTOMI $^{1}$, \\ STYLIANOS KYKALOS ${ }^{2}$, ANASTASIOS MACHAIRAS ${ }^{1}$ and DIMITRIOS SCHIZAS ${ }^{4}$
}

\author{
${ }^{1}$ Third Department of Surgery, National and Kapodistrian University of Athens, Attikon University Hospital, \\ 12462 Athens; ${ }^{2}$ Second Department of Propaedeutic Surgery, National and Kapodistrian University of Athens, \\ Laikon General Hospital, 11527 Athens; ${ }^{3}$ Second Department of Pathology, National and Kapodistrian University of \\ Athens, Attikon University Hospital, 12462 Athens; ${ }^{4}$ First Department of Surgery, National and \\ Kapodistrian University of Athens, Laikon General Hospital, 11527 Athens, Greece
}

Received December 8, 2020; Accepted April 21, 2021

DOI: $10.3892 / \mathrm{mco} .2021 .2358$

\begin{abstract}
Biliary mucinous cystic neoplasms are very rare tumors of the biliary tract with malignant potential. Preoperative diagnosis is challenging, as clinical, biochemical and radiological features are not specific. Surgical resection with negative margins is the gold standard treatment for these uncommon lesions. A 55-year-old woman presented at the Third Department of Surgery (Attikon University Hospital, Athens, Greece) with a history of mild right upper quadrant (RUQ) abdominal pain and jaundice. A 2-cm lesion in the distal common bile was identified by imaging. Following discussion in our multidisciplinary board meeting the patient underwent a pylorus preserving pancreatoduodenectomy, and histopathological examination revealed an ovarian-stromal type intraductal mucinous cystic neoplasm of the extra hepatic biliary. Since biliary mucinous cystic neoplasms are characterized by malignant transformation and high rates of recurrence, surgical resection with negative margins is the treatment of choice for both non-invasive and invasive biliary mucinous cystic neoplasms.
\end{abstract}

\section{Introduction}

Biliary mucinous cystic neoplasms (BMCNs) are rare tumors accounting for $<5 \%$ of all cystic lesions of the intrahepatic and extrahepatic biliary tree (1). BMCNs are strongly associated with female sex and present in their fourth and fifth decade of life (1). These mucus secreting or, less commonly, serous epithelium cystic lesions have been also referred to as "biliary

Correspondence to: Dr Anna Paspala, Third Department of Surgery, National and Kapodistrian University of Athens, Attikon University Hospital, Rimini 1 Street, 12462 Athens, Greece

E-mail: garoufalo@hotmail.com

Key words: biliary mucinous cystic neoplasm, mucinous cystadenoma, liver cyst, cystadenocarcinoma cystadenoma' and 'biliary cystadenocarcinoma' (2). In 2010, the World Health Organization (WHO) categorized them into non-invasive BMCNs with low-, intermediate-, or high-grade intraepithelial neoplasia and BMCNs with an invasive component $(2,3)$. Although, up to $85 \%$ of BMCNs are intrahepatic, primary BMCNs of extrahepatic biliary tract have also been described $(4,5)$. Surgical resection with negative margins is the treatment of choice, and its extent is dictated by the localization of the lesion, as such it may include hepatectomy or extended hepatectomy with Roux-en-Y reconstruction or resection of extrahepatic biliary tree, while regional lymphadenectomy is not widely recommended $(6,7)$.

We herein present a rare case of a primary extrahepatic BMCN in a middle-aged female patient who presented with mild jaundice and underwent pancreaticoduodenectomy as a therapeutic strategy.

\section{Case report}

A 55-year-old female patient presented to our department with a history of right upper quadrant (RUQ) pain associated with jaundice and weight loss. She did not report any signs or symptoms of acute cholangitis. Her physical examination was notable for a mild pain on palpation in the RUQ and for a remarkable scleral icterus. On auscultation, bowel sounds were normal, with no sign of bowel obstruction. Laboratory evaluation revealed hyperbilirubinemia, with a serum total bilirubin of $9.4 \mathrm{mg} / \mathrm{dl}$, alkaline phosphatase (ALP) of $250 \mathrm{U} / 1, \gamma$-glutamyltransferase $(\gamma \mathrm{GT})$ of $200 \mathrm{U} / 1$ and aspartate transaminase (AST) and alanine transaminase (ALT) of 50 and $40 \mathrm{U} / 1$, respectively. Moreover, regarding tumor markers, only a mild elevation of CA 19-9 (103 kU/l) was detected.Herpastmedicalhistory wasunremarkable.Thepatient underwent magnetic resonance imaging scan (MRI/MRCP), which revealed an $\sim 2 \mathrm{~cm}$ lesion located in the distal common bile duct, resulting in an excessive upstream extrahepatic and intrahepatic dilation (Fig. 1). Endoscopic retrograde cholangiopancreatography (ERCP) confirmed dilatation of the biliary tree and a large filling defect of distal CBD, while a notable amount of mucus was shown within the CBD. Given the origin 

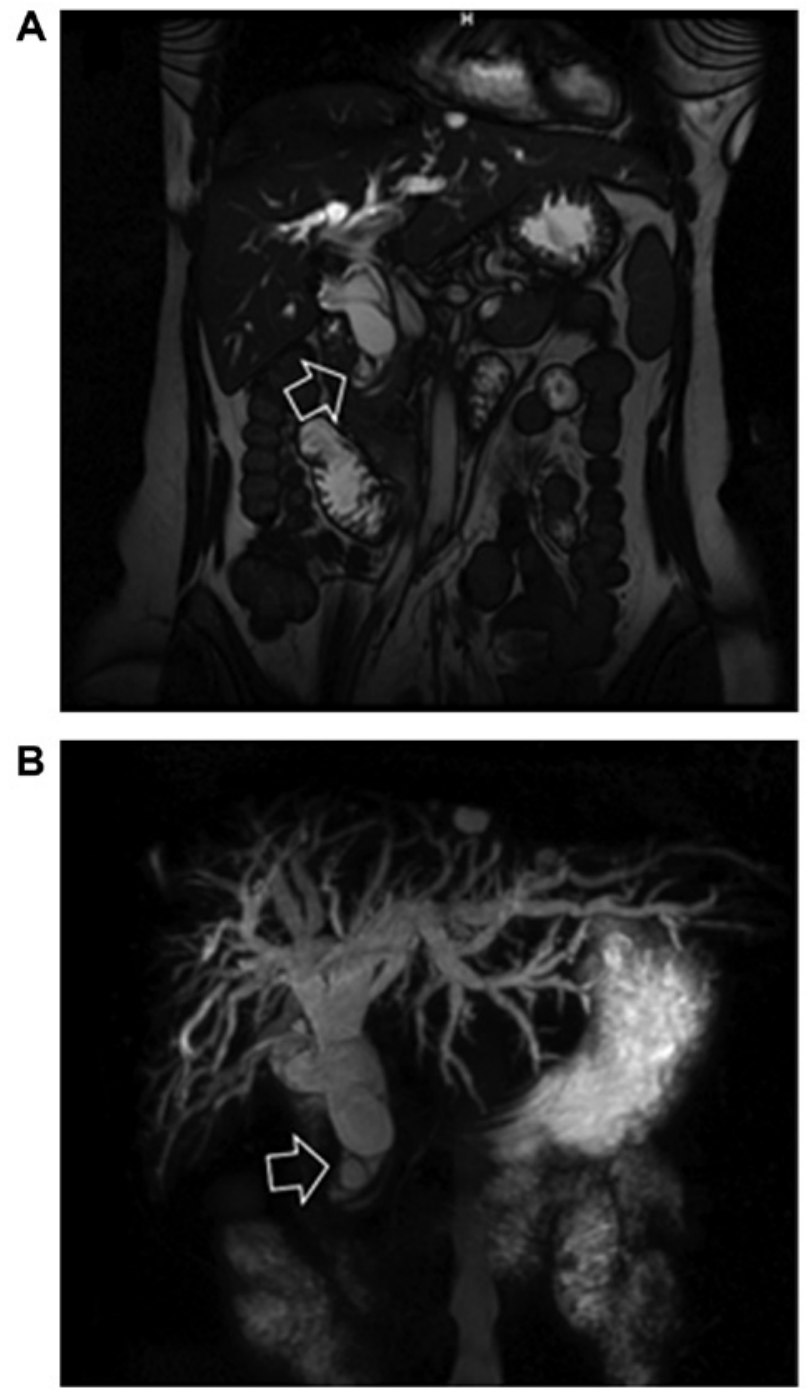

Figure 1. Preoperative MRI/MRCP of the patient. (A) MRI showing a 2-cm lesion located in the extrahepatic biliary tree (highlighted using a white arrow). (B) MRCP imaging revealing the characteristic upstream dilatation of the intrahepatic biliary tree, which was caused by the tumor (arrow). $\mathrm{MRCP}$, magnetic resonance cholangiopancreatography.

of the cystic lesion at the very distal CBD and the uncertainty about the malignant potential of the lesion, our hospital's multidisciplinary tumor board suggested radical surgical treatment.

Accordingly, the patient underwent pylorus-preserving pancreatoduodenectomy. The patient's postoperative course was complicated by a small intrabdominal abscess near the pancreaticojejunostomy, which was successfully treated with computed tomography (CT)-guided aspiration and intravenous administration of antibiotics. The rest of her postoperative course was uneventful and she was discharged on 15th postoperative day. Twenty-four months postoperatively, the patient remains disease-free in excellent clinical condition.

Histopathological examination of the resected specimen revealed the presence of a cystic neoplasm consisting of mucin producing columnar epithelium, accompanied by ovarian-type stromal elements. Minor dysplastic foci were observed throughout the neoplastic epithelium. Abundant apical mucin was evident both through Hematoxylin and eosin (H\&E) and Alcian blue histochemical staining, and the neoplastic cyst-lining epithelial cells were diffusely positive for MUC5AC (Fig. 2). More specifically, for MUC5AC immunochemistry we used Thermo Fisher Scientific antibody (REF M5-145-P) with a dilution of $1 / 150$ in room temperature. Duration of primary antibody incubation is $30 \mathrm{~min}$ (no linker) and the slides were scanned on D-Sight 200 fluo (A. Menarini Diagnostics). A hypercellular spindle-cell stromal component, exhibiting variable degrees of fibrosis accompanied the overlying epithelium without any histopathological elements indicating invasive growth. Stromal cells exhibited immunoreactivity for estrogen and progesterone receptors. Multiple calcifications along with bilirubin debris and agglomerates of basophilic microorganisms were also observed. Granulation tissue formation was evident in various areas of the tumor. The neoplasm was signed out as an intraductal mucinous cystic neoplasm of the extra hepatic biliary tract, with low-grade dysplasia.

\section{Discussion}

BMCNs of the intrahepatic and extrahepatic biliary tree, formerly known as bile duct/biliary cystadenoma and biliary cystadenocarcinoma, represent an extremely rare entity, characterized by the presence of ovarian-type stroma $(3,8)$. Besides their more common localization in the intrahepatic biliary tree, they can also develop in the extrahepatic biliary tract $(9,10)$. According to current literature, BMCNs represent less than $5 \%$ of non-parasitic hepatic cystic lesions and so far, only few case reports of these rare lesions have been published $(11,12)$. In $85-95 \%$ of cases, patients diagnosed with BMCNs are middle-aged women commonly asymptomatic or presenting with non-specific symptoms and signs, thus, the clinical diagnosis is highly incredibly challenging $(11,13,14)$. Interestingly, a case report of a young woman with intrahepatic BMCN with extrahepatic growth has also been published (10). This fact indicates the potential relationship of this entity with female-sex. The differential diagnosis of BMCNs includes hydatid cysts, simple benign liver cysts, liver abscess and mucinous cystic neoplasms with associated invasive carcinoma (11). Furthermore, similarly with our case, Pattarapuntakul et al (10) described a young woman presented with mild jaundice and impaired liver function in biochemical laboratory tests. Both our case and case published by Pattarapuntakul et al (10) underwent an MRI/MRCP and ERCP, while our case did not have an endoscopic ultrasound. Our patient underwent a Whipple procedure, and in contrast, surgical team decide to perform a left hepatectomy, because the tumor was intrahepatic with an extrahepatic growth. To our knowledge, our case is one of the few totally extrahepatic BMCNs, which treated with a pylorus-preserving Whipple procedure.

In 2000 the classification by WHO included the terms bile duct cystadenoma and cystadenocarcinoma, defined as cystic tumors, that were characterized only by mucus-secreting or, less commonly, serous epithelium (8). Moreover, ovarian-type stroma was not a mandatory criterion for histological diagnosis of these kind of lesions $(2,8)$. With regards to the current classification by WHO (2010), BMCNs are defined as epithelial cystic lesions associated with ovarian-type mesenchymal stroma (3). They are divided into non-invasive and invasive BMCNs, whilst non-invasive lesions are further classified into 


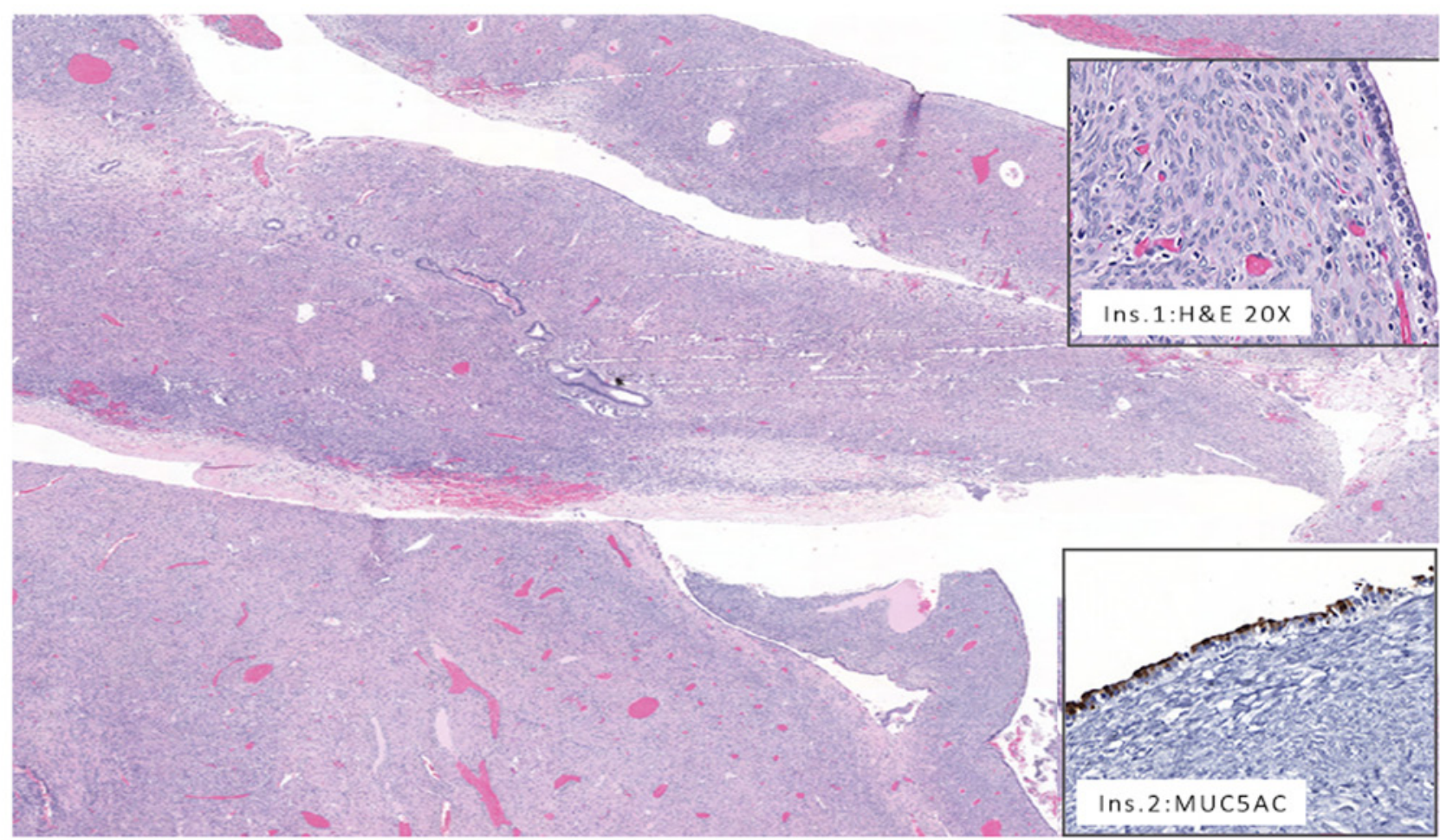

Figure 2. Histopathological evaluation of the patient. The neoplasm consisted of multiple cystic formations of various sizes accompanied by underlying hypercellular ovarian type stroma (H\&E; magnification, x10). Inset 1 (H\&E; magnification, x20). highlights that the neoplastic epithelium consisted of bland, cuboidal, columnar cells, which only occasionally exhibited minimum, low-grade dysplastic changes with disruption of normal basal cellular orientation. Inset 2 shows that MUC5AC was strongly and diffusely positive in epithelial cells (magnification, x20). Ins, inset; MUC5AC, mucin 5AC, oligomeric mucus/gel-forming.

three histological subtypes according to the grade of epithelial atypia: Low-, intermediate- and high-grade intraepithelial neoplasia $(3,15)$. Although, intrahepatic or extrahepatic BMCNs with invasive component are characterized as malignant tumors, the presence of cell atypia in the histological evaluation of BMCN suggests also significant malignant characteristics (15). On the other hand, non-invasive BMCNs with anaplastic cell morphology are defined as neoplasms with high-grade intraepithelial neoplasia (3).

Several theories have been described about the presence of ovarian-type mesenchymal stroma in liver and extrahepatic biliary tract $(16,17)$. It is known that during embryogenesis, the location of gonads, normally, is under the diaphragm and dorsally to the liver, extrahepatic biliary tree and pancreatic tail $(16,17)$. Additionally, the morphology of embryonic peritoneum in this specific location is characterized by activated cells that might reveal an evidence of relationship between gonads and developing liver, biliary tree and pancreas (16). These ectopic ovarian deposits might develop the tumors known as BMCNs by stimulating the proliferation of adjacent biliary or pancreatic ducts (18).

While differentiating BMCNs from other entities is very demanding, selecting the appropriate preoperative imaging studies in order to diagnose them is a challenging process. Preoperative imaging assessment usually includes Ultrasonography (US), CT and magnetic resonance imaging (MRI) (19). BMCNs are usually characterized by specific radiological features on CT and MRI, such as the presence of a multi-loculated and well-defined cyst, internal septations, thick capsulated cystic wall and less commonly a solid papillary component $(20,21)$. An associated significant upstream ductal dilation can be identified secondary to bile flow obstruction by the tumor, as in our case. Preoperative ERCP and percutaneous transhepatic cholangiopancreatography (PTC) are useful in demonstrating the communication between BMCNs and biliary tract, while intra-operative cholangiography might also be a useful modality in diagnosing extra-hepatic BMCNs $(22,23)$. Radiological findings such as irregular and thickened cystic wall, papillary projections, hypervascular mural solid nodules and calcifications suggest an increased suspicion of an invasive BMCN (23). However, preoperative imaging modalities cannot effectively distinguish benign from malignant BMCNs (15).

As BMCNs have a malignant potential and their preoperative differentiation from invasive BMCNs is extremely difficult, thus, the most efficient curative treatment of choice for all patients diagnosed with BMCNs and who are surgical candidates, is radical surgical resection (24). Negative margins are recommended but wide resections are not mandatory. Due to high recurrence rate, these lesions should be treated regardless of their invasiveness (25). As such, even a low-grade non-invasive BMCN should be treated with complete surgical resection (15). Enucleation with negative margins is a valid alternative option for large lesions which are located to the central liver segments and are associated with important structures such as big vessels or large bile ducts (7). Other more conservative approaches like marsupialization, internal Roux en Y drainage, aspiration and sclerosing 
agents application are associated with high rates of complications $(6,7)$. Finally, liver transplantation has been described for unresectable cases including recurrent or giant tumors $(26,27)$.

Only few cases of patients with BMCNs who were treated with chemoradiotherapy, have been described $(15,24)$. In 2009 , Vanags et al (18) reported the beneficial impact of systemic 5-fluoruracil-based chemotherapy in a patient diagnosed with recurrent and metastatic disease 41 months after the surgical resection of an invasive $\mathrm{BMCN}$. In another case, as the patient was not candidate for major hepatectomy, hepatic arterial infusion of cisplatin was used (28). The outcome of this therapeutic option was a remarkable reduction of the tumor's size from $12 \mathrm{~cm}$ to $2 \mathrm{~cm}$ (28). In addition, in a case series conducted by Läuffer et al (24), three patients received chemoradiation as the primary treatment and their 5-year overall survival rate was approximately $35 \%$.

As BMCNs are very uncommon tumors and only few studies describing these lesions have been published, it is very difficult to establish their prognosis. However, it seems to depend both on the presence or absence of invasion and the margin resection status. Moreover, Zen et al (29) reported that 24 patients with non-invasive BMCN, who were surgically treated, had a median overall survival rate of 47 months. They also described that an incomplete resection of the lesion lead to a significantly high recurrence rate (29). Almost $20 \%$ of patients with non-invasive BMCNs who had a $\mathrm{R} 1$ resection or no surgical treatment at all, can develop malignant transformation of the tumor (20). In terms of prognosis of invasive BMCNs, they have considerably better prognosis after R0 resection compared with other primary malignant hepatic lesions, such as hepatocellular carcinoma (HCC) and cholangiocarcinoma $(13,15,24)$. More precisely, the 5-year survival rate of surgically treated patients with malignant BMCNs ranges from 65 to $70 \%$, while the 5-year survival rate of $\mathrm{HCC}$ and cholangiocarcinoma is around 40 and $22 \%$, respectively (15). Interestingly, BMCNs have better prognosis in comparison to intraductal papillary mucinous neoplasms (IPMN) after surgical resection $(15,30)$. Kubota et al (30) reported that the 5-year survival rate was $100 \%$ in patients with BMCNs after R0 resection, while in patients with biliary IPMN was $84 \%$. A periodical postoperative follow-up with the already known imaging modalities, is mandatory, in order to prevent recurrence of BMCNs, especially in invasive BMCNs (31).

In conclusion, BMCNs are very rare entities, located in intrahepatic and extrahepatic biliary tree characterized by a very challenging preoperative diagnosis, as their clinical, biochemical and radiological features are not specific. Surgical resection with negative margins is the treatment of choice for both non-invasive and invasive BMCNs. Finally, due to malignant potential of these lesions and the high rates of recurrence, a meticulous postoperative surveillance is recommended.

\section{Acknowledgements}

Not applicable.

\section{Funding}

No funding was received.

\section{Availability of data and materials}

The datasets used and/or analyzed during the current study are available from the corresponding author on reasonable request.

\section{Authors' contributions}

AP, AM and DS conceived the idea and designed the study. NM, VN, SK and VD acquired, analyzed and interpreted the data. AP and DS drafted and revised the manuscript. AM and DS confirmed the authenticity of all the raw data. All authors read and approved the final manuscript.

\section{Ethics approval and consent to participate}

Not applicable.

\section{Patient consent for publication}

Written informed consent was obtained from the patient for publication of this case report and any accompanying images.

\section{Competing interests}

The authors declare that they have no competing interests.

\section{References}

1. Dixon E, Sutherland FR, Mitchell P, McKinnon G and Nayak V: Cystadenomas of the liver: A spectrum of disease. Can J Surg 44: 371-376, 2001.

2. Rodriguez RM, Barrio M, Parker ML, Saeed O, Sherman S and Ceppa EP: Mucinous cystic neoplasms of the liver: Presence of biliary communication. J Surg Case Rep 2019: rjz364, 2019.

3. Tsui WMS, Adsay NV, Crawford JM, Hurban R, Kloppel G WA: Mucinous cystic neoplasms of the liver. In: WHO Classification of Tumours of the Digestive System. 4th edition. Bosman FT, Carneiro F, Hruban RH and Theise ND, (eds). International Agency for Research on Cancer, Lyon, pp236-238, 2010.

4. Metussin A, Telisinghe P, Kok K and Chong V: Extrahepatic biliary cystadenoma: A rare cause of biliary obstruction. Oman Med J 30: 66-68, 2015.

5. Albores-Saavedra J, Córdova-Ramón JC, Chablé-Montero F, Dorantes-Heredia R and Henson DE: Cystadenomas of the liver and extrahepatic bile ducts: Morphologic and immunohistochemical characterization of the biliary and intestinal variants. Ann Diagn Pathol 19: 124-129, 2015.

6. Thomas KT, Welch D, Trueblood A, Sulur P, Wise P, Gorden DL, Chari RS, Wright JK Jr, Washington K and Pinson CW: Effective treatment of biliary cystadenoma. Ann Surg 241: 769-775, 2005.

7. Fragulidis GP,Vezakis AI,KonstantinidisCG,Chondrogiannis KK, Primetis ES, Kondi-Pafiti A and Polydorou AA: Diagnostic and therapeutic challenges of intrahepatic biliary cystadenoma and cystadenocarcinoma: A report of 10 cases and review of the literature. Int Surg 100: 1212-1219, 2015.

8. Teng XD, Hamilton SR and Aaltonen LA (eds.): World Health Organization Classification of Tumours. Pathology and Genetics of Tumours of the Digestive System. IARC Press, Lyon, 2000. Zhonghua Bing Li Xue Za Zhi 34: 544-546, 2005 (In Chinese).

9. Anand S, Chandrasekar S, Raja K and Pottakkat B: Mucinous cystic neoplasm of the liver with biliary communication: An exception to the current classification. BMJ Case Rep 12: 10-12, 2019.

10. Pattarapuntakul T, Ovartlarnporn B and Sottisuporn J: Mucinous cystic neoplasm of the liver with extrahepatic growth presenting with ascending cholangitis diagnosed by endoscopic ultrasound features: A case report. J Med Case Rep 12: 33, 2018.

11. Walt AJ: Cysts and benign tumors of the liver. Surg Clin North Am 57: 449-464, 1977. 
12. Tholomier C, Wang Y, Aleynikova O, Vanounou T and Pelletier JS: Biliary mucinous cystic neoplasm mimicking a hydatid cyst: A case report and literature review. BMC Gastroenterol 19: 103, 2019.

13. Emre A, Serin KR, Ozden I, Tekant Y, Bilge O, Alper A, Güllüoğlu $M$ and Güven $K$ : Intrahepatic biliary cystic neoplasms: Surgical results of 9 patients and literature review. World J Gastroenterol 17: 361-365, 2011.

14. Davies W, Weiland L, Batts K and Nagorney DM: Intrahepatic biliary cystadenomas with and without mesenchymal stroma. HPB 1: 141-146, 1999.

15. Simo KA, McKillop IH, Ahrens WA, Martinie JB, Iannitti DA and Sindram D: Invasive biliary mucinous cystic neoplasm: A review. HPB (Oxford) 14: 725-740, 2012.

16. Erdogan D, Kloek J, Lamers WH, Offerhaus GJA, Busch ORC, Gouma DJ and Van Gulik TM: Mucinous cystadenomas in liver: Management and origin. Dig Surg 27: 19-23, 2010.

17. Zamboni G, Scarpa A, Bogina G, Iacono C, Bassi C, Talamini G, Sessa F, Capella C, Solcia E, Rickaert F, et al: Mucinous cystic tumors of the pancreas: Clinicopathological features, prognosis, and relationship to other mucinous cystic tumors. Am J Surg Pathol 23: 410-422, 1999.

18. Vanags A, Pavars M, Prieditis P, Strumfa I, Irmejs A and Gardovskis J: Biliary cystic tumours with mesenchymal stroma. Acta Chir Latv 9: 95-99, 2010.

19. Mortelé KJ and Ros PR: Cystic focal liver lesions in the adult: Differential CT and MR imaging features. Radiographics 21: 895-910, 2001.

20. Soares KC, Arnaoutakis DJ, Kamel I, Anders R, Adams RB, Bauer TW and Pawlik TM: Cystic neoplasms of the liver: Biliary cystadenoma and cystadenocarcinoma. J Am Coll Surg 218 119-128, 2014.

21. Kim JY, Kim SH, Eun HW, Lee MW, Lee JY, Han JK and Choi BI: Differentiation between biliary cystic neoplasms and simple cysts of the liver: Accuracy of CT. Am J Roentgenol 195: $1142-1148,2010$
22. Borhani AA, Wiant A and Heller MT: Cystic hepatic lesions: A review and an algorithmic approach. Am J Roentgenol 203: 1192-1204, 2014

23. Marrone G, Maggiore G, Carollo V, Sonzogni A and Luca A Biliary cystadenoma with bile duct communication depicted on liver-specific contrast agent-enhanced MRI in a child. Pediatr Radiol 41: 121-124, 2011.

24. Läuffer JM, Baer HU, Maurer CA, Stoupis C, Zimmerman A and Büchler MW: Biliary cystadenocarcinoma of the liver: The need for complete resection. Eur J Cancer 34: 1845-1851, 1998.

25. Ohtsuka M, Shimizu H, Kato A, Yoshitomi H, Furukawa K, Tsuyuguchi T, Sakai Y, Yokosuka O and Miyazaki M: Intraductal papillary neoplasms of the bile duct. Int J Hepatol 2014: 459091, 2014.

26. Grubor NM, Colovic RB, Atkinson HD and Micev MT: Giant biliary mucinous cystadenoma of the liver. Ann Hepatol 12: 979-983, 2013.

27. Limongelli P, Pai M, Damrah O, Lauretta A, Atijosan O, Habib N and Jiao LR: Cystic tumors of the biliary tract: A complete excision is crucial. Int Surg 94: 136-140, 2009.

28. Hazanaki K, Yoshizawa K and Mori H: Hepatic arterial infusion chemotherapy of cisplatin for biliary cystadenoma. Hepatogastrenterology 46: 462-464, 1999.

29. Zen Y, Pedica F, Patcha VR, Capelli P, Zamboni G, Casaril A, Quaglia A, Nakanuma Y, Heaton N and Portmann B: Mucinous cystic neoplasms of the liver: A clinicopathological study and comparison with intraductal papillary neoplasms of the bile duct. Mod Pathol 24: 1079-1089, 2011

30. Kubota K, Nakanuma Y, Kondo F, Hachiya H, Miyazaki M, Nagino $M$, Yamamoto $M$, Is ayama $H$, Tabata $M$, Kinoshita $\mathrm{H}$, et al: Clinicopathological features and prognosis of mucin-producing bile duct tumor and mucinous cystic tumor of the liver: A multi-institutional study by the Japan Biliary Association. J Hepatobiliary Pancreat Sci 21: 176-185, 2014.

31. Ratti FA, Ferla F, Paganelli M, Cipriani FA, Aldrighetti LA and Ferla G: Biliary cystadenoma: Short-and long-term outcome after radical hepatic resection. Updates Surg 64: 13-18, 2012. 